\title{
Interstrain differences of ionotropic glutamate receptor subunits in the hippocampus and induction of hippocampal sclerosis with pilocarpine in mice
}

\author{
Q1 Endre Dobó*, Ibolya Török, András Mihály, Norbert Károly, Beáta Krisztin-Péva \\ Department of Anatomy, Faculty of Medicine, University of Szeged, Szeged, Hungary
}

\section{A R T I C L E I N F O}

\section{Article history:}

Received 18 October 2014

Received in revised form 16 January 2015

Accepted 6 February 2015

Available online xxx

\section{Keywords:}

Hippocampus

Pilocarpine

Glutamate receptor

Interstrain difference

Hippocampal sclerosis

\begin{abstract}
A B S T R A C T
Rodent strains used in epilepsy research have various neurological characteristics. These differences were suggested to be attributed to the diverse densities of the ionotropic glutamate receptor (iGluR) subunits. However, previous studies failed to find interstrain differences in the hippocampal receptor levels.

We supposed that a detailed layer-to-layer analysis of the iGluR subunits in the hippocampus might reveal strain-dependent differences in their base lines and reactions induced by pilocarpine (PILO) between two mouse strains without documented ancestors.

Levels of iGluR subunits in Balb/c and NMRI mice were compared using semiquantitative immunohistochemistry. The alterations in the neuronal circuitry were validated by neuropeptide $\mathrm{Y}$ (NPY) and neuronal nuclear antigen (NeuN) immunostainings.

Immunohistochemistry showed interstrain laminar differences in some subunits of both the control and PILO-treated animals. The seizure-induced irreversible neuronal changes were accompanied by reduced GluA1 and GluA2 levels. Their changes were inversely correlated in the individual NMRI mice by Pearson's method. Increase in NPY immunoreactivity showed positive correlation with GluA1, and negative correlation with GluA2. The NMRI strain was susceptible to PILO-induced hippocampal sclerosis, while the Balb/c animals showed resistance.
\end{abstract}

Basal levels of iGluRs differ in mouse strains, which may account for the interstrain differences in their reactions to the convulsant.

(c) 2015 Published by Elsevier B.V.

\section{Introduction}

Q2 The rodent PILO model of temporal lobe epilepsy reproduces the main neuropathological features of the human epileptic disorder therefore it has been widely used (Schauwecker, 2012; Curia et al., 2008; Winawer et al., 2007; Scharfman et al., 2001; Cavalheiro et al., 1991, 1996). The muscarinic cholinergic agonist

\footnotetext{
Abbreviations: AMPAR, AMPA receptor; DG, dentate gyrus; GC, granule cell; GluR, glutamate receptor; iGluR, ionotropic glutamate receptor; IML, internal molecular layer; KAR, kainate receptor; MC, mossy cell; MF, mossy fibre; ML, molecular layer; NMDAR, NMDA receptor; NMDAR1, NMDA receptor 1, GluN1; NeuN, neuronal nuclear antigen; NPY, neuropeptide Y; PC, pyramidal cell; PILO, pilocarpine; SGZ, supragranular zone; SL, stratum lucidum; SLM, stratum lacunosum-moleculare; SO, stratum oriens; SR, stratum radiatum.

* Corresponding author at: Department of Anatomy, Faculty of Medicine, University of Szeged, H-6724 Szeged, Kossuth L. sgt. 40, Hungary.

Tel.: +36 62 545665; fax: +36 62545707 .

E-mail address: dobo.endre@med.u-szeged.hu (E. Dobó).
}

PILO induces status epilepticus, which is followed by characteristic Q316 neuropathological changes that may lead to the appearance of 17 spontaneous recurrent seizures. After PILO-induced status epi- 18 lepticus, neuronal cell loss, gliosis and MF sprouting were observed 19 predominantly in the hippocampus (Borges et al., 2003; Turski 20 et al., 1984). The neuronal loss of hilus, one of the common 21 neuropathological features of the rodent models, was reported to 22 correlate with the development of spontaneous seizures (Mello 23 et al., 1993; Buckmaster and Dudek, 1997; Borges et al., 2003). 24

Significant increase of glutamate release is involved in status 25 epilepticus, which may play a crucial role in the development and 26 maintenance of chronic epileptic seizures (Carvalho et al., 2011; 27 Costa et al., 2004; Kovacs et al., 2003). Several studies reported that 28 PILO-induced status epilepticus also resulted in increase of gluta- 29 mate release (Cavalheiro, 1995; Smolders et al., 1997). Moreover, 30 alterations in expression and synaptic functions of glutamate 31 receptors (GluRs) were associated with glutamate excitotoxicity 32 and neuronal death (Zhang et al., 2004; Ding et al., 2007). 
GluRs may operate as canonical ion channels and/or metabotropic receptors, which elicit diverse signalling cascades. Based on the pharmacological properties and structural homology, iGluRs can be grouped into four distinct classes: AMPA receptors (AMPARs), kainate receptors (KARs), NMDA receptors (NMDARs) and $\delta$-receptors (Traynelis et al., 2010). AMPARs are characterized by very low $\mathrm{Ca}^{2+}$ permeability and fast kinetics, while NMDARs are characterized by large $\mathrm{Ca}^{2+}$ permeability and slow kinetics. While these two iGluRs are postsynaptic, the KARs are localized both preand postsynaptically and may operate as metabotropic receptors, too. The functions of the $\delta$-receptors are still unresolved. The Q4 subunit composition of the iGluRs is crucial to their function (Kortenbruck et al., 2001; Su et al., 2002).

Several studies found important species differences in the responses to convulsants (Curia et al., 2008). The species differences include various behavioural properties, e.g. susceptibility to convulsants and neuronal damages. In addition to the phylogenetic characteristics in the reactions, significant genealogical and even source-dependent intrastrain variations in seizure and cellular susceptibility were reported (Winawer et al., 2007; Portelli et al., 2009; Schauwecker, 2012). In previous experiments, we found marked individual differences between PILO-injected mice of the CFLP strain; in spite of the symptoms of acute status Q5 epilepticus, only a small fraction of the treated animals developed spontaneous recurrent seizures (Karoly et al., 2011, in preparation).

Since the hippocampal principal neurones are glutamatergic, the discrepancies between the responses of the animals to convulsants may be based on the amount and/or the composition of the functional iGluRs. However, previous studies failed to find explanation for strain differences on the hippocampal receptor level (Schauwecker, 2003; Kurschner et al., 1998). In the present experiments, we supposed that a detailed layer-to layer analysis may reveal the possible strain-dependent differences in the densities of the iGluRs.

We investigated the hippocampal distribution of iGluR subunits in two mouse strains lacking registered common ancestors by means of semiquantitative immunohistochemistry. The effects of the chemoconvulsant PILO on the densities of iGluRs were evaluated in both strains after a 2-month post-treatment period, which is thought to be sufficient for the development of spontaneous recurrent seizures (Curia et al., 2008). NPY immunohistochemistry was used to indicate the incidence of spontaneous recurrent seizures and to validate the neuropathological alterations of the hippocampal neuronal circuitry, and NeuN immunohistochemistry was applied to detect the intense neuronal loss.

Materials and methods

Animal treatment with PILO

Adult, male Balb/c and NMRI mice (25-30 g) were kept in a temperature controlled room under standard light/dark cycle, with food and water ad libitum. All experimental procedures were conducted according to the EU Directive (2010/63/ $\mathrm{EU})$ and to the Hungarian Animal Act. Specific approval of care and use of animals was obtained in advance from the Faculty Ethical Committee on Animal Experiments (University of Szeged). Animals were injected with a single intraperitoneal (i.p.) dose of PILO (Sigma-Aldrich Co., St. Louis, MO, USA) that was adjusted so as to cause at least a single occurrence of status epilepticus in only two-thirds of the animals in order to decrease the death rate. In preliminary experiments, $180 \mathrm{mg} / \mathrm{kg}$ and $195 \mathrm{mg} / \mathrm{kg}$ PILO were found to be appropriate for Balb/ c and NMRI strains, respectively. Ninety minutes after the first onset of status epilepticus, the animals were injected i.p. with diazepam (Seduxen, Gedeon Richter, Budapest, Hungary; dose: $10 \mathrm{mg} / \mathrm{kg}$ ). The NMRI strain exhibited a higher incidence rate of status epilepticus, though reacted less severely than the Balb/c strain during the PILO-induced initiation period. This protocol resulted in comparable seizure mortality: $38 \%$ in the Balb/c mice and 32\% in the NMRI strain. Post-treatment of the animals included i.p. injections with Ringer lactate solution. The control animals received the same volume of physiological saline, the solvent of PILO. The animals, which developed status epilepticus during the treatment were termed "PILOresponsive" animals.
Tissue preparation

The PILO-treated and the control animals were sacrificed 2 months after the injections. The animals were deeply anaesthetized with diethyl ether, and perfused through the ascending aorta with physiological saline, and then with 4\% formaldehyde in $0.1 \mathrm{M}$ phosphate buffer $(\mathrm{PB})$. The brains were dissected and cryoprotected overnight in $30 \%$ sucrose in $\mathrm{PB}$ at $4{ }^{\circ} \mathrm{C}$. Coronal brain sections were cut on a freezing microtome at a thickness of $24 \mu \mathrm{m}$. Section planes were selected according to the Mouse Brain Atlas of Franklin and Paxinos (1997).

\section{Immunohistochemistry}

The free-floating sections were treated with $0.5 \%$ Triton X-100 and 3\% hydrogen peroxide, then with normal swine serum (diluted: 1/10). The following primary antisera were used: rabbit anti-NPY (Abcam, Cambridge, UK, dilution: 1/10,000): mouse anti-NeuN (Chemicon, Temecula, CA, USA, dilution: 1/8000); rabbit antiGluA1 (Millipore, Temecula, CA, USA, 1/500); mouse anti-GluA2 (Chemicon, dilution: 1/200); rabbit anti-GluA2/3 (Chemicon, dilution: 1/400); monoclonal rabbit anti-GluK2 (clone: EPR6307; Abcam, dilution: 1/3000); mouse anti-NMDAR1 (Abcam, dilution: 1/5000). The sections were incubated under continuous agitation at room temperature overnight. After washing, the sections were incubated with the appropriate biotinylated secondary antibody (Jackson ImmunoResearch, West Grove, PA, USA, dilution: 1/400) for $60 \mathrm{~min}$, and finally with peroxidase-labelled streptavidin (Jackson ImmunoResearch, dilution: 1/1000) for $60 \mathrm{~min}$. The sites of immunoreaction were visualized with diaminobenzidine in the absence or presence of nickel (Adams, 1981). At one particular series of a given immunostaining for assessment, all the sections were incubated in aliquots from the same solutions of either the immunoreagents or the chromogens synchronously for exactly the same time. The same number of sections of the control and PILOresponsive animals of both strains was incubated in the same volume of the solutions.

Image analysis

Pictures were taken with an image-capture system (Olympus DP50) attached to an Olympus BX-50 microscope (Soft Imaging System GmbH, Münster, Germany). Image analysis was performed with Adobe Photoshop 7 (Adobe Systems Incorporated, San Jose, CA, USA). A researcher blind to the experimental conditions of the animals measured the pixel density of immunostained images. Briefly: through use of the "marquee" tool, 8-12 circular, $0.1 \mathrm{~mm}$ diameter areas were selected in adjacent positions inside the hippocampal layers. The average of 10 background determinations (carried out near the layers in unstained neuropilcontaining sites) was subtracted from the average pixel densities measured within the hippocampal layers. Differences between the corresponding hippocampal regions of the control and PILO-responsive, but non-sclerotic animals were assessed by using the unpaired one-tailed Student's $t$-test. Pearson's correlation analysis was used to evaluate the relationship between the optical densities of different hippocampal layers. Data were analyzed and plotted with the aid of GraphPad 4.0 (GraphPad Software, Inc., CA, USA).

\section{Results}

\section{NPY immunohistochemistry}

According to previous data (Scharfman and Gray, 2006), only a few, small NPY-immunoreactive neurones were scattered throughout the hippocampus of the control animals (Fig. 1A). These interneurones displayed short non-varicose branches. Dramatic increase in hippocampal NPY synthesis was reported to be a diagnostic tool to confirm the incidence of spontaneous recurrent seizures (Sperk et al., 1992; Borges et al., 2003; Scharfman and Gray, 2006). After the PILO treatment the NPY immunoreactivity greatly increased in the whole area of the dentate gyrus (DG) and in the stratum lucidum (SL) of CA3 in every PILO-responsive mouse (Fig. 1B), irrespective of the strains.

The vast majority of the increased immunostaining was localized in the synaptic fields of the MFs. We did not observe the NPY staining in the perikarya of dentate granule cells (GCs). Apart from the heavy staining of the MF areas, NPY-immunoreactive puncta were seen in the thin supragranular zone (SGZ) within the internal molecular layer (IML) of the DG. The immunoreactivity increased in the molecular layer (ML) too, but in much less extent than in the areas supplied by MFs. Furthermore, the NPYimmunoreactive cells in the CA1 region displayed stronger staining 

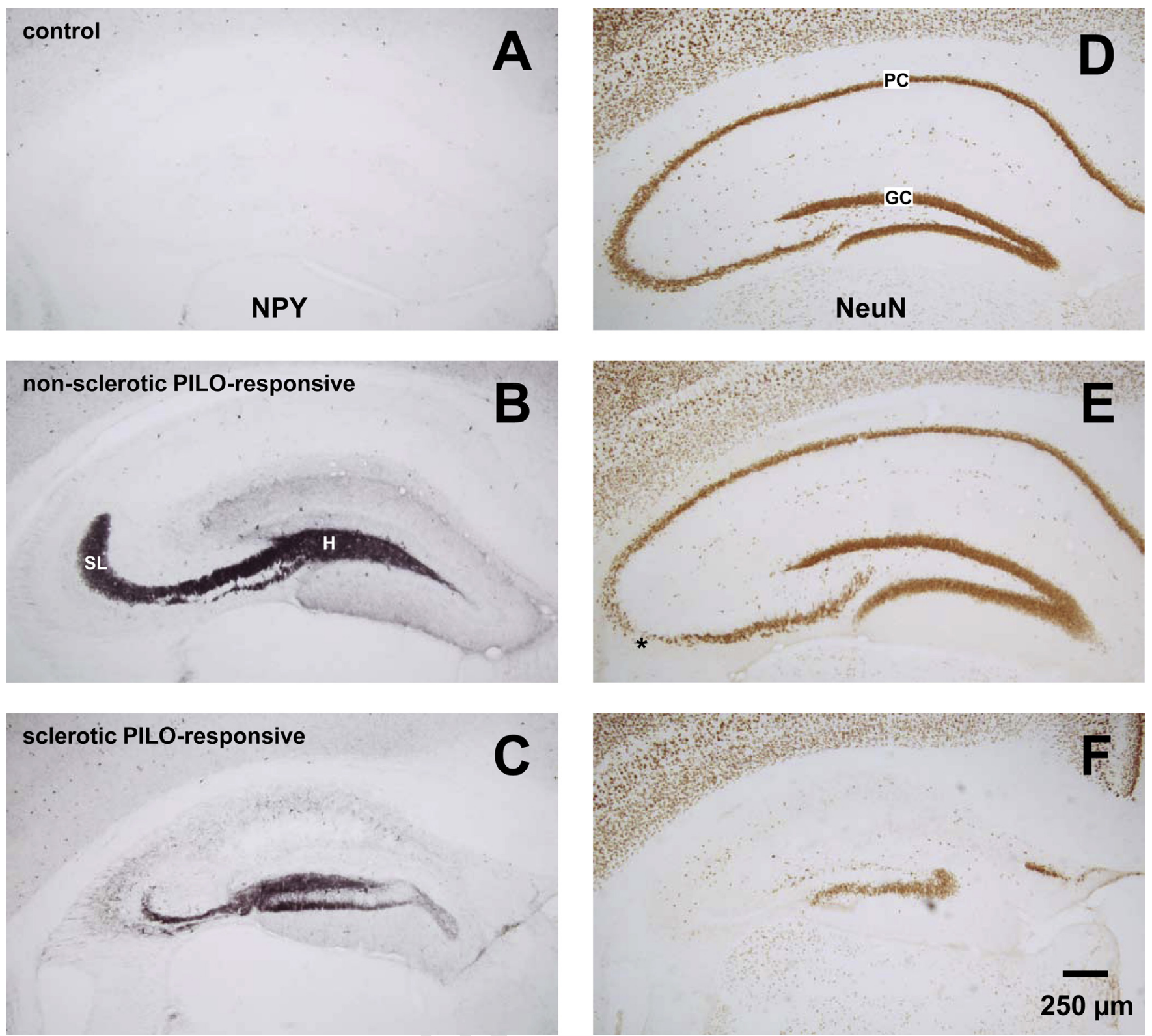

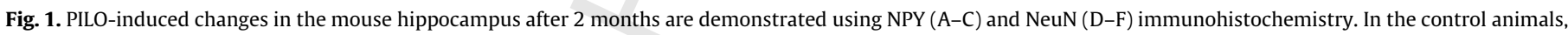

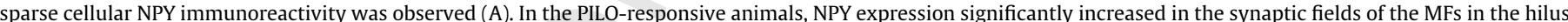

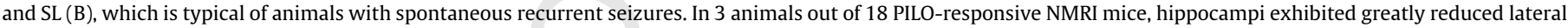

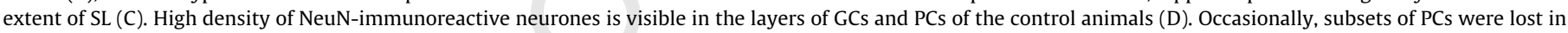

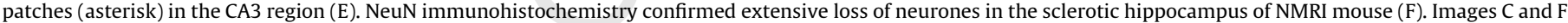
were taken from the same animal. SL: stratum lucidum; H: hilus; GC: granule cell; PC: pyramidal cell. Scale bar: $250 \mu$ m.

in PILO-responsive animals. Newly formed immunopositive processes were not detected in this region.

In the NMRI strain, but not in the Balb/c mice, 3 animals out of 18 PILO-responsive mice developed hippocampal sclerosis (Fig. 1C). In the sclerosed hippocampi, the lateral extent and the width of SL in the CA3a were dramatically reduced, but the NPY staining was strong. In these three animals, the hilus of the DG was also strongly labelled similarly to other PILO-responsive animals of the NMRI and Balb/c strains.

\section{NeuN immunohistochemistry}

Neuronal loss was checked by means of NeuN immunostaining in the PILO-responsive animals. In 3 out of the 7 PILO-responsive Balb/c mice, the number of pyramidal cells (PCs) in the CA3a and
CA3b subregions was slightly reduced (Fig. 1D and E). No considerable loss of PCs was observed in the CA1, CA2 and CA3c regions. By comparing the subsequent NPY- and NeuN-immunostained sections of the same Balb/c animals, no correlation was detected between the extent of NPY immunoreactivity and the degree of cell loss.

In the NMRI strain, the cell loss was more pronounced, 8 out of the 18 PILO-responsive mice displayed patchy neuronal loss in the PC layer of CA 1 and CA 3 regions. In 3 out of 18 PILO-responsive animals, the marked loss of PCs extended from the CA3a/b to the CA3c subregion (Fig. 1F). The PILO-responsive animals that displayed the loss of the entire NeuN-immunoreactive CA3 PC populations were referred as to sclerotic in this study. The paired comparisons of the NPY- and NeuN-immunostained sections revealed that the NPY-immunoreactive SL was greatly shortened in

Please cite this article in press as: Dobó, E., et al., Interstrain differences of ionotropic glutamate receptor subunits in the hippocampus and induction of hippocampal sclerosis with pilocarpine in mice. J. Chem. Neuroanat. (2015), http://dx.doi.org/10.1016/ j.jchemneu.2015.02.002 
197 the animals with this pattern of neuronal damage (Fig. 1C and F). 198 The absence of CA3c PCs and the characteristic shortage of NPYimmunoreactive SL differentiate the aforementioned 3 mice from the other PILO-responsive individuals. Beside the loss of CA3 PCs, in the two sclerotic mice, the superior blade of the GC layer was damaged to a large extent (Fig. 1F). Because of the distorted hippocampal structure of the sclerotic mice, the consistent laminar analysis of the iGluR subunits was not possible.

\section{AMPAR immunohistochemistry}

AMPAR antibodies to GluA1, GluA2, GluA2/3 provided similar immunostaining in the consecutive sections (Fig. 2). The strongest immunostaining was observed with the GluA1 antibody (Fig. 2A), while the GluA2/3 antibody gave the weakest staining (Fig. 2E). The antibodies stained mainly neuropil: the most intense staining was experienced in the stratum oriens (SO) and stratum radiatum (SR) of CA1 region. The least intense staining was found in the hilus of the DG and in the SL of CA3. GluA2 and GluA2/3 antibodies also stained several multipolar neurones in the hilus (Fig. 2C and E), which were supposedly mossy cells (MCs) (Tang et al., 2005). It is worth noting that the layer-to-layer comparisons of the semiquantitative data of the two strains revealed significant density differences in the GluA2 immunoreactivities of the DG. The ML and the hilus of the NMRI mice showed lower values compared to the Balb/c mice ( $-23 \%$ and $-79 \%$, respectively, empty columns in Fig. 3).

PILO-treatment resulted in remarkable changes in the immunoreactivity, the extent of which was analyzed in some of the hippocampal layers by means of semiquantitative immunohistochemistry (Table 1, Fig. 3). The density of the GluA1 immunoreactivity decreased in every hippocampal layer (Fig. 2B), except the CA1 SR of the NMRI mice. In all other layers, very similar changes were observed in both strains. The most significant reductions were found in the dentate hilus $(-72 \%$ for Balb/c and $-69 \%$ for NMRI).

The GluA2 immunostaining density also decreased in both strains (Fig. 2D). The intensity changes in the layers were two- or three-fold higher in the Balb/c strain than in the NMRI strain. The highest reduction of the GluA2 immunoreactivity was found in the synaptic field of the MFs in both strains. The statistical analysis of the GluA2/3 immunohistochemical results (Fig. 2F) showed largely similar alterations. The lowest density values and the highest degree of reduction of the optical densities were found in the hilus of the DG in the Balb/c and NMRI mice $(-58 \%$ and $-45 \%$, respectively). The density of the hilar immunopositive cells significantly reduced in both mouse strains $(-29 \%$ for Balb/c and $-62 \%$ for NMRI).

\section{GluK2 immunohistochemistry}

The application of the rabbit monoclonal antibody for the detection of the low affinity GluK2 KAR subunit resulted in a staining pattern very similar to that of the AMPAR antibodies in the hippocampus (Fig. 2G). In the control animals, strong immunoreactivity was observed in the hippocampus. Weak immunostaining was found in the pyramidal and the granular layers, in the hilus and in the SL of CA3 (Fig. 2G). The layer-to-layer comparisons of the data from the two strains revealed significant intensity differences in the GluK2 immunoreactivities: the ML and the hilus of the NMRI strain exhibited higher density values than the Balb/c mice $(+22 \%$ and $+79 \%$, respectively, empty columns in Fig. 3 ).

After PILO treatment, the intensity of the GluK2 immunoreactivity increased in the hippocampus (Fig. $2 \mathrm{H}$ ). An increase in the immunostaining density was found in every hippocampal layer of the Balb/c mice: the highest increase was observed in the hilus
(+43\%; Table 1). Opposite alteration, the decrease of the GluK2 density was observed in the hilar region of the NMRI mice $(-27 \%$; Table 1). Intensity increases were also significant in the SR of CA1 and the stratum lacunosum-moleculare (SLM) of CA3 in the Balb/c strain $(+23 \%$ and $+15 \%$, respectively). In the NMRI mice similar, but less increase was observed in those layers ( $+13 \%$ in SR, $+8 \%$ in SLM). The ML of the Balb/c mice showed $+13 \%$ intensity increase, while no alteration was measured in the ML of the NMRI mice. In both strains, no significant changes were detected in the staining intensity of the SL (Table 1 ).

\section{GluN1 immunohistochemistry}

GluN1 immunohistochemistry revealed a laminar staining pattern in the control hippocampus, which was similar to the AMPAR immunostaining (Fig. 2I). In contrast to the AMPAR antibodies, the GluN1 antibody did not label neurones in any of the examined areas. The most intense neuropil staining was found in the SO and the SR. The staining of these layers was increasing towards the subiculum. Moderate immunostaining was experienced in the SLM and the SM, while the weakest staining was observed in the hilus, the SL of CA3 and in the pyramidal and the granular layers. The staining in the hilus was almost nil, therefore no measurements were done there. On the other hand, the SGZ displayed a relatively strong labelling, which was evaluated in PILO-treated mice.

PILO treatment exerted measurable effects on the GluN1 immunostaining in the hippocampal layers of the two examined mouse strains (Fig. 2J, Table 1). In the Balb/c specimens the intensity of the staining in the SR and in the SLM of CA1 were significantly decreased $(-32 \%$ and $-36 \%$, respectively, Table 1$)$. The PILO treatment did not cause modification in the immunostaining density of the overall ML of the DG. However, in the close vicinity of the GC layer, that is in the SGZ our semiquantitative method revealed a significant intensity decrease $(-16 \%)$. In the NMRI mice the only significant change, an increase (+29\%) was measured in the SLM of CA1.

\section{Correlation analysis}

The optical density data of the immunohistochemical stainings in the hippocampal layers of the non-sclerotic PILO-responsive NMRI mice were subjected to correlation analysis. The pairwise comparisons within the individual animals revealed several significant relationships between the densities of the receptor subunits and NPY staining.

A set of relationships concerns the dendritic field of GCs in the ML and their axon terminal field in the SL. While in the ML considerably decreased GluA1 immunoreactivity was found, in the SL markedly increased NPY immunoreactivity was measured. The pairwise correlations yielded a significant negative correlation value $(-0.636)$ between the pixel densities of the GluA1 and NPY, i.e. the higher density of the NPY immunoreactivity coincided with the more decrease of the GluA1 immunoreactivity, compared to the average values of the control animals (Fig. 4).

Opposite changes were found when densities of NPY in the SL and GluA2 in the ML were subjected to pairwise correlations. Although, marked increase and decrease in the immunoreactivities were found for NPY and GluA2, respectively, the appropriate correlations revealed a positive correlation value $(+0.462)$ between the pixel densities of the NPY and GluA2, i.e. the higher increase in the immunoreactivity for NPY coincided with the less decrease (relatively elevated level) of the GluA2 staining (Fig. 4).

Pairwise comparisons of the GluA1 and GluA2 immunoreactivities in the ML confirmed the previous data analysis. Although, 

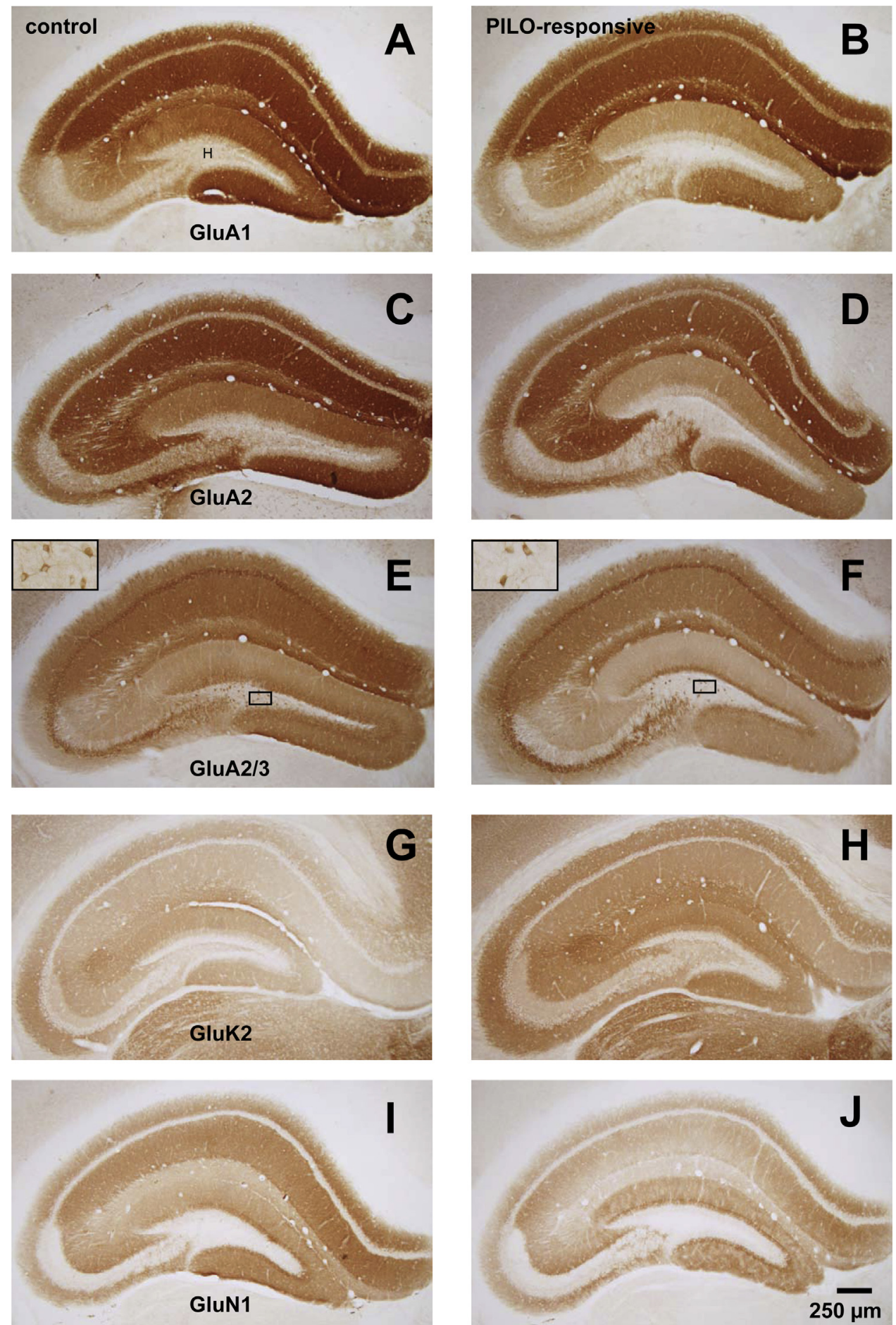

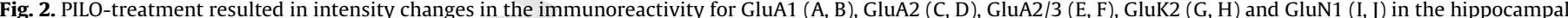

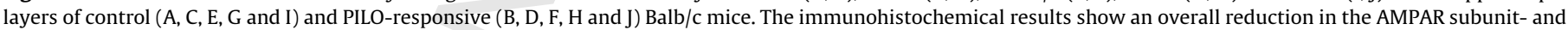

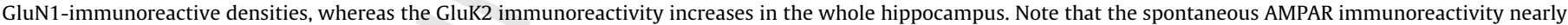

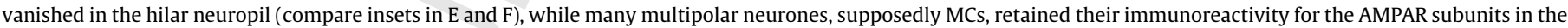
hilus. H: hilus. Scale bar: $250 \mu \mathrm{m}$.

significant decreases of both staining intensities were found, the pairwise correlation showed a negative correlation value $(-0.445)$ between the pixel densities of the GluA1 and GluA2, i.e. the stronger immunoreaction with GluA1 seemed to be significantly related with the weaker immunoreactivity for GluA2, which denoted inversely proportional changes of the GluA1 and GluA2 immunoreactivities (Fig. 4). Decreased GluA1 immunoreactivity in the hilus was found to be inversely correlated with the decrease in the immunoreactivity for GluK2 in the same layer (Pearson's correlation value: -0.557 ), i.e. the less the hilar GluA1 immunoreactivity decreased, the more the GluK2 immunoreactivity reduced in the hilus. The pairwise correlations between the changes in the GluR subunits within the hippocampal layers showed also that in the SL the less increased NPY-immunoreactive layer was coincided with the more decreased GluA2-immunoreactive one (Pearson's correlation value: +0.467$)$.

Please cite this article in press as: Dobó, E., et al., Interstrain differences of ionotropic glutamate receptor subunits in the hippocampus and induction of hippocampal sclerosis with pilocarpine in mice. J. Chem. Neuroanat. (2015), http://dx.doi.org/10.1016/ j.jchemneu.2015.02.002 

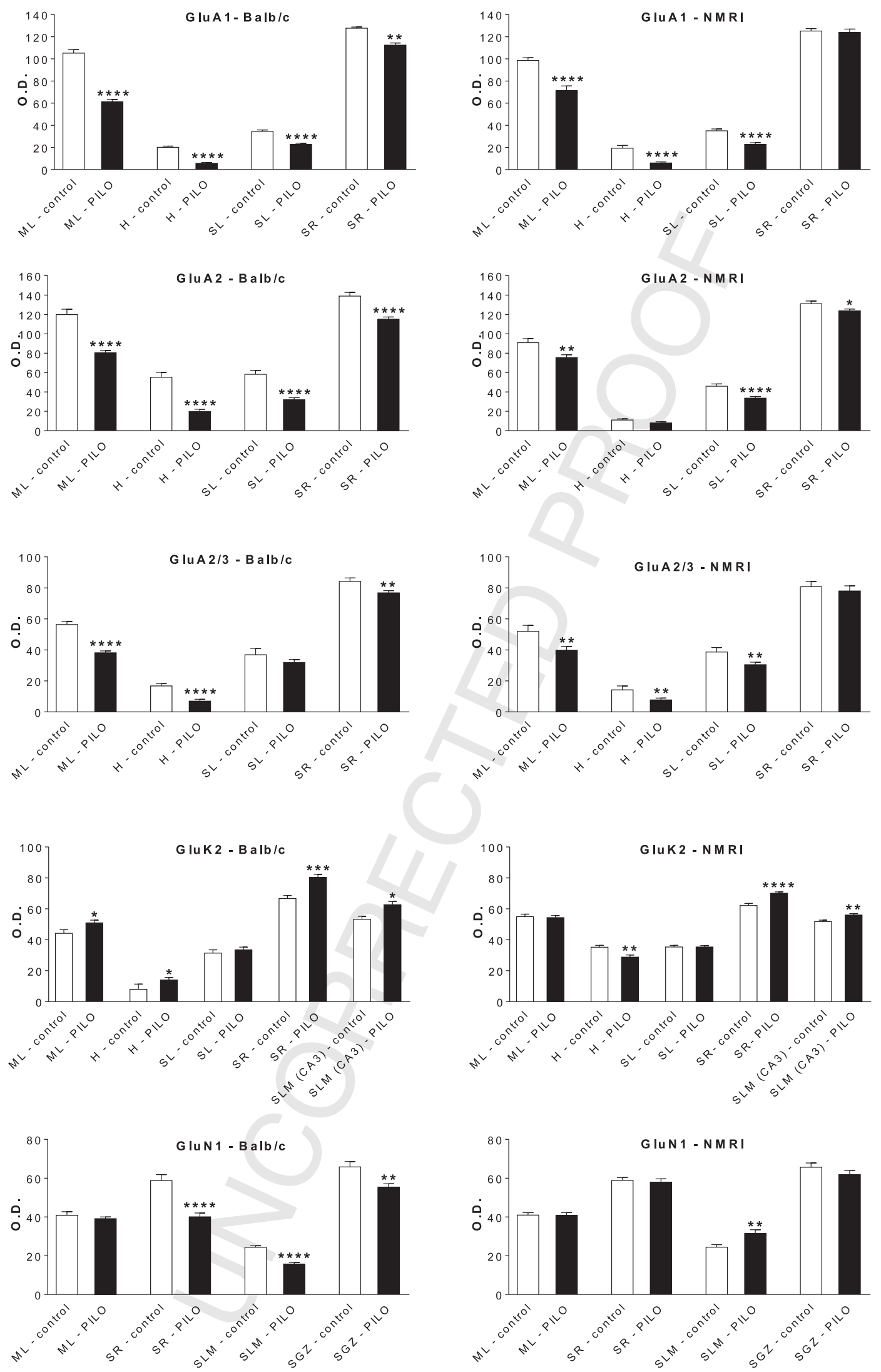

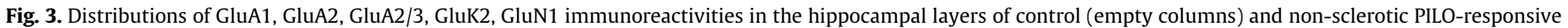

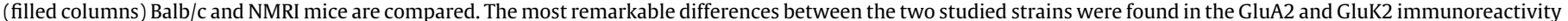

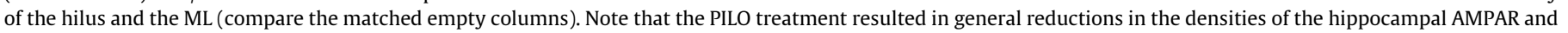

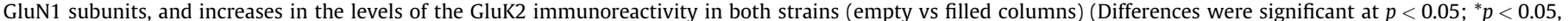

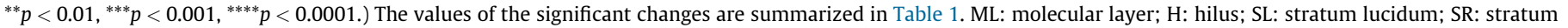
radiatum; SLM: stratum lacunosum-moleculare; SGZ: supragranular zone.

Please cite this article in press as: Dobó, E., et al., Interstrain differences of ionotropic glutamate receptor subunits in the hippocampus and induction of hippocampal sclerosis with pilocarpine in mice. J. Chem. Neuroanat. (2015), http://dx.doi.org/10.1016/ j.jchemneu.2015.02.002 
Table 1

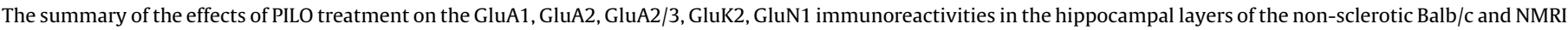
mice. The changes of immunoreactivities are expressed in percent.

\begin{tabular}{|c|c|c|c|c|c|c|c|c|}
\hline & Animal strain & SR & SLM & SLM (CA3) & ML & SGZ & $\mathrm{H}$ & SL \\
\hline \multirow[t]{2}{*}{ GluA1 } & Balb/c & $-12^{* *}$ & & & $-42^{* * * * *}$ & & $-72^{* * * *}$ & $-34^{* * * *}$ \\
\hline & NMRI & -1 & & & $-28^{* * * *}$ & & $-69^{* * * *}$ & $-35^{* * * *}$ \\
\hline \multirow[t]{2}{*}{ GluA2 } & Balb/c & $-17^{* * * *}$ & & & $-33^{* * * *}$ & & $-64^{* * * *}$ & $-45^{* * * * *}$ \\
\hline & NMRI & $-6^{*}$ & & & $-18^{* *}$ & & -32 & $-27^{* * * *}$ \\
\hline \multirow[t]{2}{*}{ GluA2/3 } & Balb/c & $-9^{* *}$ & & & $-32^{* * * * *}$ & & $-58^{* * * *}$ & -14 \\
\hline & NMRI & -3 & & & $-21^{* *}$ & & $-45^{* *}$ & $-23^{* *}$ \\
\hline \multirow[t]{2}{*}{ GluK2 } & $\mathrm{Balb} / \mathrm{c}$ & $+23^{* * *}$ & & $+15^{*}$ & $+13^{*}$ & & $+43^{*}$ & +6.5 \\
\hline & NMRI & $+13^{* * * *}$ & & $+8^{* * *}$ & -3 & & $-27^{* * *}$ & +1 \\
\hline \multirow[t]{2}{*}{ GluN1 } & Balb/c & $-32^{* * * *}$ & $-36^{* * * *}$ & & -4 & $-16^{* *}$ & & \\
\hline & NMRI & -2 & $+29^{* *}$ & & -0.4 & -6 & & \\
\hline
\end{tabular}

The values of significance are indicated as follows:

${ }^{*} p<0.05$.

$p<0.01$.

$p<0.001$

$p<0.0001$.

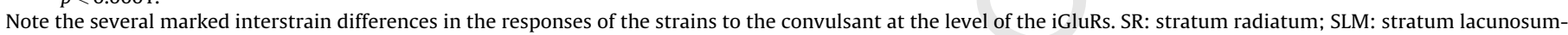
moleculare; ML: molecular layer; SGZ: supragranular zone; H: hilus; SL: stratum lucidum.

\section{The sclerotic hippocampus of the NMRI mice}

The PILO-responsive NMRI mice, but not the Balb/c mice, showing greatly enhanced NPY immunoreactivity in the hilus and SL, were divided into two groups on the basis of the severity of the NeuN-immunoreactive cell loss in the CA3. In this study, the arbitrary distinction between the groups was the presence or the absence of PCs in the CA3c subfield in the non-sclerotic PILOresponsive and the sclerotic mice, respectively. In the non-sclerotic PILO-responsive group, CA3c PCs persisted even in those cases where a complete segment of CA3a/b vanished (Fig. 5). In this group, the NPY-immunoreactive SL seemingly remained unchanged. However, in the 3 sclerotic mice out of 18 PILOresponsive ones, the damage to the CA3 PCs was coincided with the drastic loss of the NPY enhanced area (Figs. 1C and 5).

Immunohistochemistry for the iGluRs in the sclerotic mice revealed robust changes in the distributions of all studied subunits, when compared with those in the non-sclerotic PILO-responsive mice. In the sclerotic animals, large areas lost their corresponding immunoreactivities partially or even completely. Very strong coincidence was observed in the severity of the density reductions in the immunoreactivities for GluA1, GluK2, GluN1 and NeuN. The areas with highly reduced immunoreactivity involved the CA1

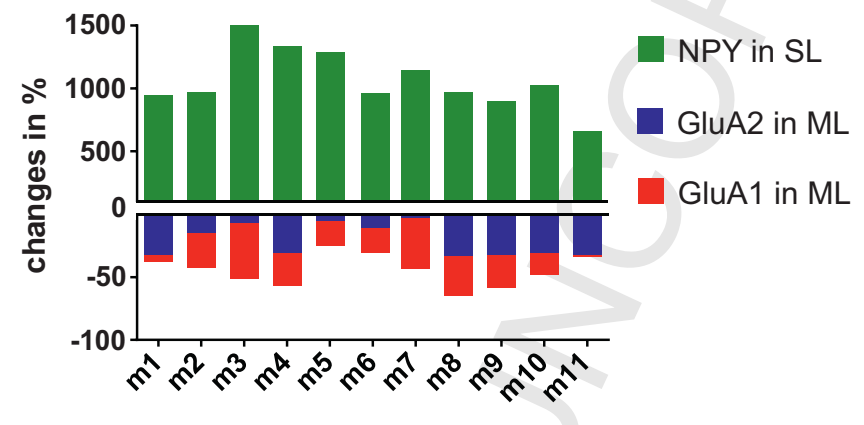

individual mice

Fig. 4. Correlations between the changes in the immunostainings of NPY in the terminal field of the MF (SL) and iGluR subunits in the dendritic field of GCs (ML) were probed in the individual non-sclerotic PILO-responsive NMRI mice by the Pearson's correlation analysis. Bidirectional relationships were found between the NPY and the iGluRs immunoreactivities; the more intense immunoreactivity for NPY coincided with the more and the less decreases in immunoreactions with GluA1 and GluA2, respectively, the changes in the GluA1 and GluA2 immunostainings were inversely proportional. Correlation values of pairwise comparisons were -0.636 between NPY and GluA1, 0.462 between NPY and GluA2, -0.445 between GluA1 and GluA2. SL: stratum lucidum; ML: molecular layer. region, including all of its layers (Fig. 5). The damage to the SO was more extended than that of the SR in the same section. The more CA1 PCs were lost, the larger areas in the SR disappeared from the immunostaining. In the case of the considerable loss of GCs, the ML of the DG lying towards the damaged SR was also greatly affected. It is worth noting that in the apparently intact areas within the ML, the immunoreactivity for GluK2 seemed to be slightly increased (Fig. 5L), whereas immunoreactivities for GluA1 and GluN1 in the corresponding areas in the subsequent sections were significantly reduced.

\section{Discussion}

Hippocampal sclerosis and axonal sprouting

Temporal lobe epilepsy is the most common type of epilepsy in adults, which is frequently associated with hippocampal sclerosis, which is a complex histopathological manifestation of neuronal cell loss and aberrant fibre sprouting. Several lines of evidence suggest the vulnerability of excitatory MCs (Scharfman and Myers, 2012), PCs (Wasterlain et al., 1993; Borges et al., 2003) and the vulnerability of some inhibitory neurones (Houser and Esclapez, 1996) to the seizures. The question whether the loss of cells contributed to the epileptogenesis or the cell loss was the consequence of the repeated seizures has not been answered reliably. The surviving GCs and inhibitory neurones react to convulsions and cell death with excessive fibre/axonal sprouting (Curia et al., 2008; Levesque and Avoli, 2013). There is a general notion that the activity of GCs increases in spite of earlier suggestions that GCs are hyperinhibited and remain relatively quiet during spontaneous seizures (Harvey and Sloviter, 2005).

GCs do not only sprout but also change their chemotype during 06387 the chronic seizure (Gutiérrez, 2003). Several experiments proved that tonic-clonic seizures evoked with electrical kindling (Rizzi et al., 1993), kainate (Gruber et al., 1994; Sperk et al., 1992) or PILO (Lurton and Cavalheiro, 1997) result in the appearance of strong and long-lasting NPY immunoreactivity in MFs. The persistent NPY immunoreactivity in hippocampal sclerosis indicated the importance of this chemotype change in the maintenance of the seizures and/or in the survival of GCs.

\section{Mouse strain differences}

Various animal models offer suitable strategies for the investigation of complex neurological disorders such as epilepsy. Extrapolation of the results from one species and/or strain to 

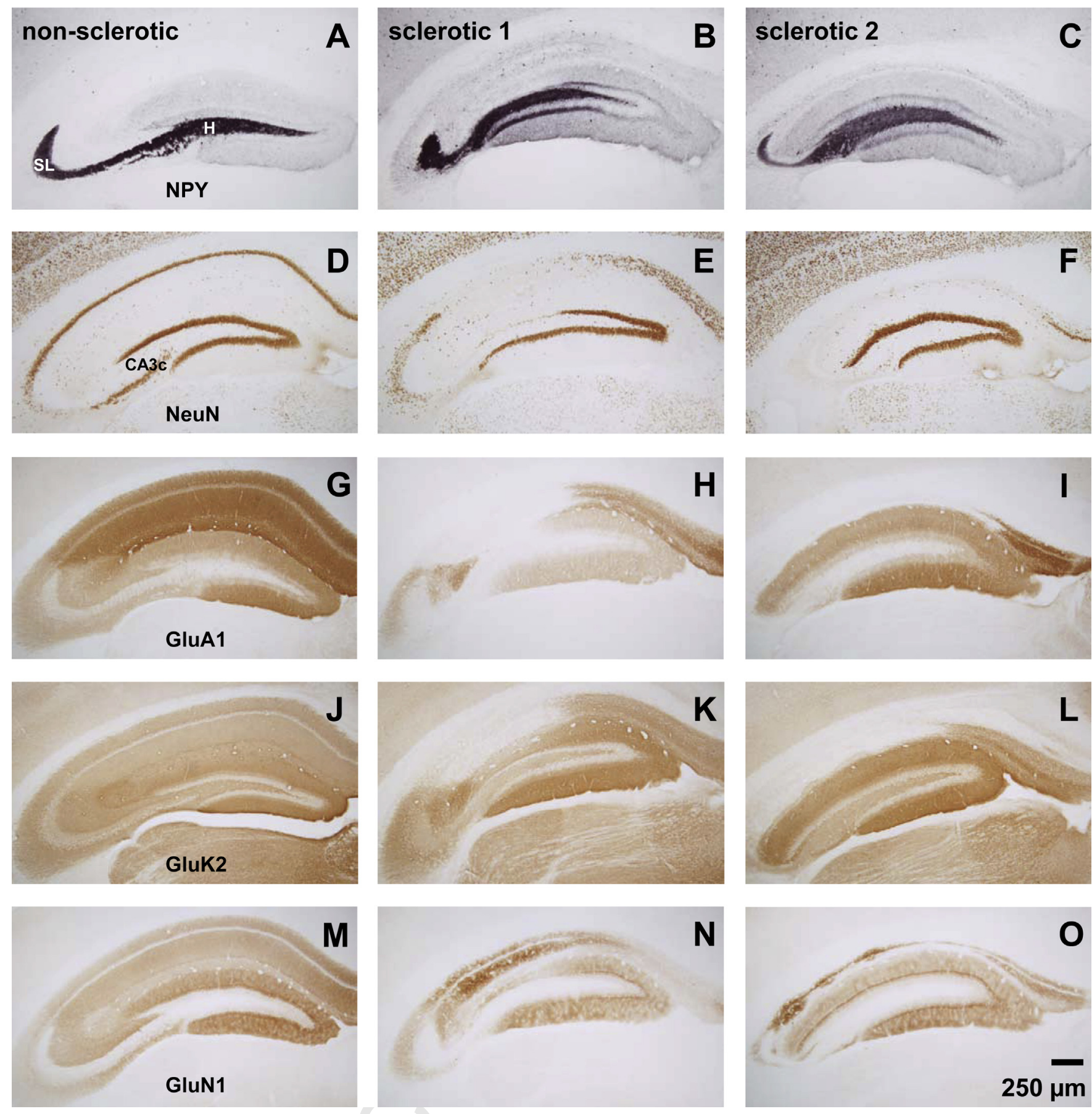

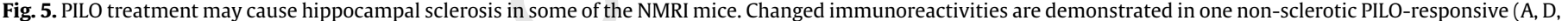

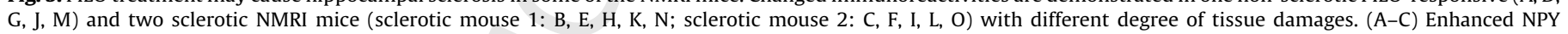

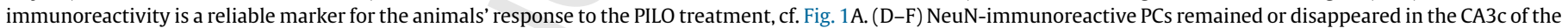

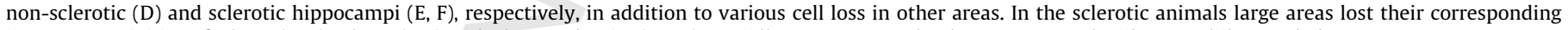
immunoreactivities of GluA1 (G-I), GluK2 (J-L) and GluN1 subunits (M-O) partially or even completely. SL: stratum lucidum; H: hilus. Scale bar: $250 \mu m$.

others, is fairly questionable, since the rodents demonstrate significant species-, strain- and even intrastrain differences in the susceptibility to convulsive agents and the consequences of seizures (Curia et al., 2008; Portelli et al., 2009).

Certain rodent strains exhibiting high resistance to chemically induced status epilepticus did not undergo degeneration or cell damage in spite of similar seizure severity (Schauwecker and Steward, 1997). In our study, Balb/c and NMRI strains were compared for their chronic responses to the chemoconvulsant PILO. According to the historical records (Beck et al., 2000; Chia et al., 2005) about the origins of these strains, no common progenitors were found. The immunohistochemical staining revealed considerable hippocampal differences, though the NPY immunoreactivity was equally intense in the hilus and SL after 2month post-treatment period. It is noteworthy that we recently reported our results about the effects of PILO treatment on a third mouse strain, CFLP (Karoly et al., 2011), which was thought not to share progenitors with the other two strains of this study (Beck et al., 2000). Strikingly, in contrast to Balb/c and NMRI strains, all individuals of which suffered from the status epilepticus showed marked NPY immunoreactivity in the field of the MFs, a considerable portion of the PILO-treated CFLP mice failed to

Please cite this article in press as: Dobó, E., et al., Interstrain differences of ionotropic glutamate receptor subunits in the hippocampus and induction of hippocampal sclerosis with pilocarpine in mice. J. Chem. Neuroanat. (2015), http://dx.doi.org/10.1016/ j.jchemneu.2015.02.002 
exhibit either marked NPY immunoreactivity or ectopic MF sprouting in the SGZ.

The strain-adjusted doses of PILO that were applied did not result in visible neuronal loss in the NeuN immunostained preparations of $57.1 \%$ of the Balb/c and $55.5 \%$ of the NMRI. In the rest of the animals, the $\mathrm{CA} 3 \mathrm{a} / \mathrm{b}$ subregions lost the neurones in both strains in various degrees. An important difference between the two studied strains was the specific elimination of CA3c PCs from 17\% of the PILO-responsive NMRI, whereas these cells seemed to remain intact in the Balb/c mice. This difference is in line with several papers reporting that (1) Balb/crelated strains (Balb/cJ and Balb/cByJ) were found to be resistant to PILO-induced status epilepticus for noticeable neuronal loss (Schauwecker, 2012), and (2) the descendants of the so called Swiss mice, a separate genealogical line (Beck et al., 2000), including the NMRI strain, were found to undergo severe Q7 damage to the CA3 PCs (Turski et al., 1984; Riban et al., 2002; Tang et al., 2005).

\section{AMPA receptors}

The efficacy of the AMPAR-associated glutamatergic neurotransmission depends on the density, the stoichiometry of the combining subunits, the modifications of the subunits (Russo et al., 2013). Receptor-binding studies measuring AMPARs in the hippocampal homogenates from seizure-prone (DBA/2J) and seizure-resistant (C57BL/6J) mice did not find detectable interstrain differences (Frandsen et al., 1987; Kurschner et al., 1998). No strain-dependent differences of the GluR subunits in the intact hippocampi of mice were found with immunohistochemical methods, either (Schauwecker, 2003). However, our semiquantitative layer-to-layer comparisons of the AMPAR subunits showed interstrain differences between the age-matched control animals in the GluA2 but not in the GluA1. The differences were confined to the DG; the hilus contained less GluA2 $(-79 \%)$ in the NMRI mice compared to the Balb/c. Coincidentally, the NMRI strain received more amount of PILO than the Balb/c strain to obtain equal number of PILO-responsive animals. Since the hilar GluA2 immunoreactivity is accounted for by the MCs, the remarkably higher density of the $\mathrm{Na}^{+}$-permeable GluA2 immunoreactivity in the Balb/c may serve as an explanation for its higher vulnerability to PILO than the NMRI strain.

After the PILO treatment, our AMPAR immunohistochemical results showed an overall reduction in the density of this iGluR type in the non-sclerotic PILO-responsive hippocampus. It was indicated earlier that the experimental inhibition of AMPARs can prevent long-term increases in seizure susceptibility and seizureinduced neuronal injury (Koh et al., 2004). Thus, the significant decrease of AMPARs of the PILO-responsive hippocampus suggested an extensive attenuation of excitatory response to glutamate.

In the Balb/c mice, the decreases of the GluA1 and GluA2 subunits were comparable; their layer-by-layer ratios seemed unaltered. The general reduction of the AMPAR density and the absence of the change in the ratio of the GluA1 and GluA2 subtypes indicated an intrinsic neuroplastic mechanism for counterbalancing the increased excitability of hippocampus.

In the NMRI mice, the degree of the changes was less marked than in the Balb/c strain, though the loss of the PCs and MCs was more pronounced in the NMRI than in the Balb/c, resulting in proneness of NMRI to hippocampal sclerosis, but not the Balb/c. Besides, the MCs were more vulnerable to the PILO treatment in the NMRI than in the Balb/c (62\% and $29 \%$, respectively). This comparison also indicates that the general reduction in the AMPAR level is not directly correlated with the loss of the principal neurons.
The changes of the subtypes in the NMRI strain were examined by means of Pearson's correlation analysis in order to see the relationships between the GluA1 and GluA2 subunits in the individual animals. The results showed that the robust reductions in the GluA1 and GluA2 densities ( $-28 \%$ and $-18 \%$, respectively) in the ML were composed of significant $(-0.445)$, inversely proportional changes of these two GluR subunits; even though the densities of both subunits decreased.

The correlation analysis between the changes of GluA1 and GluA2 in the ML and the changes of NPY in the SL pointed to significant tendencies, too. GCs, which may receive strong synchronized nerve impulses in the ML, and terminate in the SL, can be appropriate links between these three parameters. In the ML, the relatively less reduced density of the GluA2 may render the AMPAR less permeable the membrane for $\mathrm{Ca}^{2+}$. Thereby, the $\mathrm{Ca}^{2+}-$ operated $\mathrm{K}^{+}$channels may be less activated, which are the key elements for the afterhyperpolarization. The shorter duration of afterhyperpolarization may less prevent the GCs from the intense input. This susceptibility of the cells for the seizure-like activity could be compensated by two mechanisms. On one hand, the density of the GluA1 is more reduced. On the other hand, the density of the inhibitory NPY is more elevated in the terminal field of GCs. This tentative explanation may be in an agreement with some authors' notion that the increased NPY immunoreactivity in the SL of the mice is a reliable indicator of the incidence of recurrent seizures (Sperk et al., 1992; Borges et al., 2003; Scharfman and Gray, 2006).

Low-affinity kainate receptor: GluK2

KARs are present on both sides of the synapse, where they play distinct and diverse roles (Huettner, 2003; Lerma, 2003; Fernandes et al., 2009; Sihra et al., 2014). In our experiments, the GluK2 subunit was chosen to represent the distribution of the KARs by means of immunohistochemistry. This subunit was supposed to play a central role in the formation of presynaptic and postsynaptic KARs (Wenthold et al., 1994; Contractor et al., 2001). In the control animals, interstrain differences were confined to the DG: the ML and the hilus of the NMRI mice contained $+22 \%$ and $+79 \%$ more immunoreactivity, respectively, than those of the Balb/c strain. Investigation of GluK2-overexpressing and knockout animals suggested that the presence of hippocampal GluK2 promotes seizure activity (Mulle et al., 1998; Telfeian et al., 2000).

Although the densities of the immunohistochemistry for the AMPARs were found to be generally decreased in the non-sclerotic PILO-responsive animals in both mouse strains, immunostaining for the GluK2 was ambiguously altered between the two strains and between the hippocampal layers within the given strain. In Balb/c mice, the decrease of AMPARs was accompanied by the increase of KAR density in the DG. In the NMRI mice, AMPAR decreases were not followed by opposite KAR alterations.

The diverse changes in the GluK2 levels within the individual hippocampal layers cannot be interpreted reliably on the basis of the data available in the literature (Vincent and Mulle, 2009). The GluK2 mRNA is mainly expressed in the glutamatergic principal cells of the hippocampus (Paternain et al., 2000). Presynaptic GluK2-containing KARs can modulate glutamate release not only via ionotropic but also via metabotropic modes (RodriguezMoreno and Sihra, 2007). Furthermore, glutamate may exert bimodal effect on its own release in a concentration dependent manner on certain presynaptic elements (Ruiz and Kullmann, 2012). The net effects of the GluK2-associated changes on the spontaneous recurrent seizures may also be affected by the GABAergic interneurones, which may receive glutamatergic inputs, and are involved in the regulation of the activity of the hippocampal principal cells (Christensen et al., 2004). 
NMDARs colocalize with AMPARs to form the functional synaptic unit at virtually all central synapses, where the NMDARs can modulate glutamatergic neurotransmission postsynaptically by generating long-lasting $\mathrm{Ca}^{2+}$ influx and depolarization. The functional channels are heteromeric consisting of the obligatory GluN1 and the associated other subunits of any of the NR2A-D subtypes (Garcia-Gallo et al., 2001).

Interstrain differences were also experienced after the comparison of the GluN1 immunohistochemical results of the two studied strains. In the Balb/c mice, marked decrease was detected in the apical dendritic field of CA1 PCs: reductions were found in the SR, where the Schaffer collaterals terminate, and in the SLM $(-32 \%$ and $-36 \%$, respectively) where many fibres of the temporo-ammonic pathway innervate the distal dendritic branches of PCs. The reduction in the CA1 may result from the downregulation of the NMDARs, which was also reported in electroconvulsive seizures (Park et al., 2014).

The similar degrees of reductions in the SR and SLM may be accounted for by either the lack of precise membrane trafficking of the NMDAR subunits to input-specific sites along the dendritic tree, or the similar degrees of involvements of the putative epileptic excitations at CA1 PCs along the perforant path fibre-MFSchaffer collateral axis and the temporo-ammonic pathway. The GluN1 reduction in the SGZ could be attributed to the appearance of the ectopic MFs in this sublayer, as indicated by many previous reports (Buckmaster, 2012; Pierce et al., 2005).

GluN1 immunohistochemical staining of the NMRI hippocampi resulted in a significant increase in the SLM of CA1, which may contribute to the increased susceptibility of this strain to the PILOinduced hippocampal sclerosis.

\section{Conclusions}

1. Comparison of the laminar distribution of the iGluR subunits in two mouse strains (Balb/c and NMRI) with no documented common ancestors revealed some interstrain differences. The most remarkable differences between the two strains were found in GluA2 and GluK2 immunoreactivity of the hilus and the ML, which may indicate the involvement of MCs in the interstrain differences in their predisposition to PILO-induced neuronal alterations.

2. PILO-induced status epilepticus resulted in significantly different degrees of changes in the laminar immunoreactivitiy for the iGluR subunits in the two strains. The alterations of the neuronal circuitry showed bidirectional relationships with the inversely correlated changes of the GluA1 and GluA2 levels in the DG of the individual non-sclerotic NMRI mice.

3. The PILO treatment caused sclerotic hippocampi in some NMRI mice, whilst Balb/c animals seemed to be more resistant to hippocampal neuronal death. The big strain difference suggests that the researchers have to be careful in choosing the suitable strains as the model animals for studying temporal lobe epilepsy, since the genetic divergence can highly determine the diverse disposition to hippocampal sclerosis.

\section{Author contributions}

ED and IT designed research; IT, ED and NK performed research; IT, ED and BKV analyzed data; ED, IT and AM wrote the paper.

\section{Acknowledgement}

613 Q8 Grant: TÁMOP 4.2.2-A-11/1/KONV-2012-0052.
Adams, J.C., 1981. Heavy metal intensification of DAB-based HRP reaction product J. Histochem. Cytochem. 29 (6), 775.

Beck, J.A., Lloyd, S., Hafezparast, M., Lennon-Pierce, M., Eppig, J.T., Festing, M.F. et al., 2000. Genealogies of mouse inbred strains. Nat. Genet. 24 (1), $23-25$.

Borges, K., Gearing, M., McDermott, D.L., Smith, A.B., Almonte, A.G., Wainer, B.H. et al., 2003. Neuronal and glial pathological changes during epileptogenesis in the mouse pilocarpine model. Exp. Neurol. 182 (1), 21-34.

Buckmaster, P.S., 2012. Mossy fiber sprouting in the dentate gyrus. In: Noebels, J.L., Avoli, M., Rogawski, M.A., Olsen, R.W., Delgado-Escueta, A.V. (Eds.), Jasper's Basic Mechanisms of the Epilepsies, Bethesda, MDfourth ed.

Buckmaster, P.S., Dudek, F.E., 1997. Neuron loss, granule cell axon reorganization, and functional changes in the dentate gyrus of epileptic kainate-treated rats. J. Comp. Neurol. 385 (3), 385-404.

Carvalho, A.S., Torres, L.B., Persike, D.S., Fernandes, M.J., Amado, D., Naffah-Mazzacoratti Mda, G., et al., 2011. Neuroprotective effect of pyruvate and oxaloacetate during pilocarpine induced status epilepticus in rats. Neurochem. Int. 58 (3), $385-390$.

Cavalheiro, E.A., 1995. The pilocarpine model of epilepsy. Ital. J. Neurol. Sci. 16(1-2) $33-37$.

Cavalheiro, E.A., Leite, J.P., Bortolotto, Z.A., Turski, W.A., Ikonomidou, C., Turski, L., 1991. Long-term effects of pilocarpine in rats: structural damage of the brain triggers kindling and spontaneous recurrent seizures. Epilepsia 32 (6), 778-782.

Cavalheiro, E.A., Santos, N.F., Priel, M.R., 1996. The pilocarpine model of epilepsy in mice. Epilepsia 37 (10), 1015-1019.

Chia, R., Achilli, F., Festing, M.F., Fisher, E.M., 2005. The origins and uses of mouse outbred stocks. Nat. Genet. 37 (11), 1181-1186.

Christensen, J.K., Paternain, A.V., Selak, S., Ahring, P.K., Lerma, J., 2004. A mosaic of functional kainate receptors in hippocampal interneurons. J. Neurosci. 24 (41), 8986-8993.

Contractor, A., Swanson, G., Heinemann, S.F., 2001. Kainate receptors are involved in short- and long-term plasticity at mossy fiber synapses in the hippocampus. Neuron 29 (1), 209-216.

Costa, M.S., Rocha, J.B., Perosa, S.R., Cavalheiro, E.A., Naffah-Mazzacoratti, M.da G 2004. Pilocarpine-induced status epilepticus increases glutamate release in rat hippocampal synaptosomes. Neurosci. Lett. 356 (1), 41-44.

Curia, G., Longo, D., Biagini, G., Jones, R.S., Avoli, M., 2008. The pilocarpine model of temporal lobe epilepsy. J. Neurosci. Methods 172 (2), 143-157.

Ding, S., Fellin, T., Zhu, Y., Lee, S.Y., Auberson, Y.P., Meaney, D.F., et al., 2007. Enhanced astrocytic $\mathrm{Ca}^{2+}$ signals contribute to neuronal excitotoxicity after status epilepticus. J. Neurosci. 27 (40), 10674-10684.

Fernandes, H.B., Catches, J.S., Petralia, R.S., Copits, B.A., Xu, J., Russell, T.A., et al., 2009. High-affinity kainate receptor subunits are necessary for ionotropic but not metabotropic signaling. Neuron 63 (6), 818-829.

Frandsen, A., Belhage, B., Schousboe, A., 1987. Differences between seizure-prone and non-seizure-prone mice with regard to glutamate and GABA receptor binding in the hippocampus and other regions of the brain. Epilepsy Res. 1 (2), 107-113.

Franklin, K.B.J., Paxinos, G., 1997. The Mouse Brain in Stereotaxic Coordinates. San Diego, Academic Press.

Garcia-Gallo, M., Renart, J., Diaz-Guerra, M., 2001. The NR1 subunit of the N-methylD-aspartate receptor can be efficiently expressed alone in the cell surface of mammalian cells and is required for the transport of the NR2A subunit. Biochem. J. 356 (Pt. 2), 539L 547.

Gruber, B., Greber, S., Rupp, E., Sperk, G., 1994. Differential NPY mRNA expression in granule cells and interneurons of the rat dentate gyrus after kainic acid injection. Hippocampus 4 (4), 474-482.

Harvey, B.D., Sloviter, R.S., 2005. Hippocampal granule cell activity and c-Fos expression during spontaneous seizures in awake, chronically epileptic, pilocarpine-treated rats: implications for hippocampal epileptogenesis. J. Comp. Neurol. 488 (4), 442-463.

Houser, C.R., Esclapez, M., 1996. Vulnerability and plasticity of the GABA system in the pilocarpine model of spontaneous recurrent seizures. Epilepsy Res. 26 (1) 207-218.

Huettner, J.E., 2003. Kainate receptors and synaptic transmission. Prog. Neurobiol 70 (5), 387-407.

Karoly, N., Mihaly, A., Dobo, E., 2011. Comparative immunohistochemistry of synaptic markers in the rodent hippocampus in pilocarpine epilepsy. Acta Histochem. 113 (6), 656-662.

Koh, S., Tibayan, F.D., Simpson, J.N., Jensen, F.E., 2004. NBQX or topiramate treatment after perinatal hypoxia-induced seizures prevents later increases in seizure-induced neuronal injury. Epilepsia 45 (6), 569-575.

Kortenbruck, G., Berger, E., Speckmann, E.J., Musshoff, U., 2001. RNA editing at the $\mathrm{Q} / \mathrm{R}$ site for the glutamate receptor subunits GLUR2, GLUR5, and GLUR6 in hippocampus and temporal cortex from epileptic patients. Neurobiol. Dis. 8 (3), 459-468.

Kovacs, A., Mihaly, A., Komaromi, A., Gyengesi, E., Szente, M., Weiczner, R., et al. 2003. Seizure, neurotransmitter release, and gene expression are closely related in the striatum of 4-aminopyridine-treated rats. Epilepsy Res. 55 (1-2), 117-129.

Kurschner, V.C., Petruzzi, R.L., Golden, G.T., Berrettini, W.H., Ferraro, T.N., 1998 Kainate and AMPA receptor binding in seizure-prone and seizure-resistant inbred mouse strains. Brain Res. 780 (1), 1-8.

Lerma, J., 2003. Roles and rules of kainate receptors in synaptic transmission. Nat. Rev. Neurosci. 4 (6), 481-495.
615

616

617

618

619

620

621

623

624

625

626

627

628
629

630

631

632

633

634

635
636

637

638

639

640

641
642

643

644

645

646
647

648

649

650

651

652

654

655

656

657
658

659

660

661

662

663
664

665

666

667

668

670

671

672

673

674
675

676

677

678
679

680

681

682

683

684
685

686

687

688

689

691

692

693

694

695

696

698 
G Model

CHENEU 1316 1-11

E. Dobó et al./Journal of Chemical Neuroanatomy $x x x$ (2015) $x x x-x x x$

11

699

700

701

703

704

705

707

708

709

710

711

713

714

715

717

718

719

720

721

723

724

725

726

728

729

730

731

733

734

735

736

738

739

740

741

742
Levesque, M., Avoli, M., 2013. The kainic acid model of temporal lobe epilepsy. Neurosci. Biobehav. Rev. 37 (10 Pt. 2), 2887L 2899.

Lurton, D., Cavalheiro, E.A., 1997. Neuropeptide-Y immunoreactivity in the pilocarpine model of temporal lobe epilepsy. Exp. Brain Res. 116 (1), 186-190.

Mello, L.E., Cavalheiro, E.A., Tan, A.M., Kupfer, W.R., Pretorius, J.K., Babb, T.L., et al., 1993. Circuit mechanisms of seizures in the pilocarpine model of chronic epilepsy: cell loss and mossy fiber sprouting. Epilepsia 34 (6), 985-995.

Mulle, C., Sailer, A., Perez-Otano, I., Dickinson-Anson, H., Castillo, P.E., Bureau, I., et al., 1998. Altered synaptic physiology and reduced susceptibility to kainateinduced seizures in GluR6-deficient mice. Nature 392 (6676), 601-605.

Park, H.G., Yu, H.S., Park, S., Ahn, Y.M., Kim, Y.S., Kim, S.H., 2014. Repeated treatment with electroconvulsive seizures induces HDAC2 expression and down-regulation of NMDA receptor-related genes through histone deacetylation in the rat frontal cortex. Int. J. Neuropsychopharmacol. 17 (9), 1487-1500.

Paternain, A.V., Herrera, M.T., Nieto, M.A., Lerma, J., 2000. GluR5 and GluR6 kainate receptor subunits coexist in hippocampal neurons and coassemble to form functional receptors. J. Neurosci. 20 (1), 196-205.

Pierce, J.P., Melton, J., Punsoni, M., McCloskey, D.P., Scharfman, H.E., 2005. Mossy fibers are the primary source of afferent input to ectopic granule cells that are born after pilocarpine-induced seizures. Exp. Neurol. 196 (2), 316-331.

Portelli, J., Aourz, N., De Bundel, D., Meurs, A., Smolders, I., Michotte, Y., et al., 2009. Intrastrain differences in seizure susceptibility, pharmacological response and basal neurochemistry of Wistar rats. Epilepsy Res. 87 (2-3), 234-246.

Rizzi, M., Monno, A., Samanin, R., Sperk, G., Vezzani, A., 1993. Electrical kindling of the hippocampus is associated with functional activation of neuropeptide Ycontaining neurons. Eur. J. Neurosci. 5 (11), 1534-1538.

Rodriguez-Moreno, A., Sihra, T.S., 2007. Metabotropic actions of kainate receptors in the CNS. J. Neurochem. 103 (6), 2121-2135.

Ruiz, A.J., Kullmann, D.M., 2012. Ionotropic receptors at hippocampal mossy fibers: roles in axonal excitability, synaptic transmission, and plasticity. Front. Neural Circuits 6, 112.

Russo, I., Bonini, D., Via, L.L., Barlati, S., Barbon, A., 2013. AMPA receptor properties are modulated in the early stages following pilocarpine-induced status epilepticus. Neuromol. Med. 15 (2), 324-338.

Scharfman, H.E., Gray, W.P., 2006. Plasticity of neuropeptide $Y$ in the dentate gyrus after seizures, and its relevance to seizure-induced neurogenesis. EXS (95), $193-211$.

Scharfman, H.E., Myers, C.E., 2012. Hilar mossy cells of the dentate gyrus: a historical perspective. Front. Neural Circuits 6, 106.

Scharfman, H.E., Smith, K.L., Goodman, J.H., Sollas, A.L., 2001. Survival of dentate hilar mossy cells after pilocarpine-induced seizures and their synchronized burst discharges with area CA3 pyramidal cells. Neuroscience 104 (3), 741-759.

Schauwecker, P.E., 2003. Differences in ionotropic glutamate receptor subunit expression are not responsible for strain-dependent susceptibility to excitotoxin-induced injury. Brain Res. Mol. Brain Res. 112 (1-2), 70-81.
Schauwecker, P.E., 2012. Strain differences in seizure-induced cell death following pilocarpine-induced status epilepticus. Neurobiol. Dis. 45 (1), 297-304.

Schauwecker, P.E., Steward, O., 1997. Genetic determinants of susceptibility to excitotoxic cell death: implications for gene targeting approaches. Proc. Natl. Acad. Sci. U. S. A. 94 (8), 4103-4108.

Sihra, T.S., Flores, G., Rodriguez-Moreno, A., 2014. Kainate receptors: multiple roles in neuronal plasticity. Neuroscientist 20 (1), 29-43.

Smolders, I., Khan, G.M., Lindekens, H., Prikken, S., Marvin, C.A., Manil, J., et al., 1997. Effectiveness of vigabatrin against focally evoked pilocarpine-induced seizures and concomitant changes in extracellular hippocampal and cerebellar glutamate, gamma-aminobutyric acid and dopamine levels, a microdialysis-electrocorticography study in freely moving rats. J. Pharmacol. Exp. Ther. 283 (3) 1239-1248.

Sperk, G., Marksteiner, J., Gruber, B., Bellmann, R., Mahata, M., Ortler, M., 1992. Functional changes in neuropeptide $\mathrm{Y}$ - and somatostatin-containing neurons induced by limbic seizures in the rat. Neuroscience 50 (4), 831-846.

Tang, F.R., Chia, S.C., Zhang, S., Chen, P.M., Gao, H., Liu, C.P., et al., 2005. Glutamate receptor 1-immunopositive neurons in the gliotic CA1 area of the mouse hippocampus after pilocarpine-induced status epilepticus. Eur. J. Neurosci. 21 (9), 2361-2374.

Telfeian, A.E., Federoff, H.J., Leone, P., During, M.J., Williamson, A., 2000. Overexpression of GluR6 in rat hippocampus produces seizures and spontaneous nonsynaptic bursting in vitro. Neurobiol. Dis. 7 (4), 362-374.

Traynelis, S.F., Wollmuth, L.P., McBain, C.J., Menniti, F.S., Vance, K.M., Ogden, K.K., et al., 2010. Glutamate receptor ion channels: structure, regulation, and function. Pharmacol. Rev. 62 (3), 405-496.

Turski, W.A., Cavalheiro, E.A., Bortolotto, Z.A., Mello, L.M., Schwarz, M., Turski, L., 1984. Seizures produced by pilocarpine in mice: a behavioral, electroencephalographic and morphological analysis. Brain Res. 321 (2), 237-253.

Vincent, P., Mulle, C., 2009. Kainate receptors in epilepsy and excitotoxicity. Neuroscience 158 (1), 309-323.

Wasterlain, C.G., Fujikawa, D.G., Penix, L., Sankar, R., 1993. Pathophysiological mechanisms of brain damage from status epilepticus. Epilepsia 34 (Suppl. 1), S37-S53.

Wenthold, R.J., Trumpy, V.A., Zhu, W.S., Petralia, R.S., 1994. Biochemical and assembly properties of GluR6 and KA2, two members of the kainate receptor family, determined with subunit-specific antibodies. J. Biol. Chem. 269 (2), 1332-1339.

Winawer, M.R., Makarenko, N., McCloskey, D.P., Hintz, T.M., Nair, N., Palmer, A.A. et al., 2007. Acute and chronic responses to the convulsant pilocarpine in DBA 2J and A/J mice. Neuroscience 149 (2), 465-475.

Zhang, G., Raol, Y.S., Hsu, F.C., Brooks-Kayal, A.R., 2004. Long-term alterations in glutamate receptor and transporter expression following early-life seizures are associated with increased seizure susceptibility. J. Neurochem. 88 (1), $91-101$.

744

745

747

748

749

751

752

754
755

756

758

759

761

762
763

764
765

766
767

768

769
770

771

772
773

774
775

776

777

779

780

781
782

783

784

786

787
788

Please cite this article in press as: Dobó, E., et al., Interstrain differences of ionotropic glutamate receptor subunits in the hippocampus and induction of hippocampal sclerosis with pilocarpine in mice. J. Chem. Neuroanat. (2015), http://dx.doi.org/10.1016/ j.jchemneu.2015.02.002 